\title{
Arthroscopic superior capsule reconstruction using autologous fascia lata and biceps tendon augmentation
}

\section{Chen-Heng Hsu}

Bone and Joint Research Center, Department of Orthopaedic Surgery, Chang Gung Memorial HospitalLinkou and Chang Gund University College of Medicine

\section{Chih-Hao Chiu}

Bone and Joint Research Center, Department of Orthopaedic Surgery, Chang Gung Memorial HospitalLinkou and Chang Gung University Colleg of Medicine

\section{Chun-Jui Weng}

Bone and Joint Research Center, Department of Orthopaedic Surgery, Chang Gung Memorial HospitalLinkou and Chang Gung University College of Medicine

\section{Kuo-Yau Hsu}

Bone and Joint Research Center, Department of Orthopaedic Surgery, Chang Gung Memorial HospitalLinkou and Chang Gung University College of Medicine

\section{Yi-Sheng Chan}

Bone and Joint Resarch Center, Department of Orthopaedic Surgery, Chang Gung Memorial HospitalLinkou and Chang Gung University College of Medicine

\section{Alvin Chao-Yu Chen ( $\square$ alvinchen@cgmh.org.tw)}

Bone and Joint Research Center, Department of Orthopaedic Surgery, Chang Gung Memorial HospitalLinkou, and Chang Gung University College of Medicine https://orcid.org/0000-0003-0111-490X

\section{Technical note}

Keywords: superior capsule reconstruction (SCR), long head of biceps tendon (LHBT), fascia lata, irreparable, rotator cuff, acromiohumeral distance

Posted Date: June 4th, 2020

DOI: https://doi.org/10.21203/rs.3.rs-32772/v1

License: (c) (i) This work is licensed under a Creative Commons Attribution 4.0 International License. Read Full License 
Version of Record: A version of this preprint was published at Arthroscopy Techniques on June 1st, 2021. See the published version at https://doi.org/10.1016/j.eats.2021.02.004. 


\section{Abstract \\ Background}

While arthroscopic superior capsule reconstruction (SCR) has been recently introduced to treat irreparable rotator cuff tear with encouraging outcomes, graft options and fixation remain debated. The purpose of this article is to describe a novel technique and present preliminary results in using long head of biceps tendon (LHBT) as augmentation for SCR with fascia lata autograft.

\section{Methods}

Retrospective analysis was performed on 18 patients undergoing arthroscopic SCR with LHBT augmentation for irreparable rotator cuff tear between October 2017 and November 2019. Preoperative radiographic survey confirmed massive rotator cuff tear with moderate to severe muscle fatty infiltration and availability of LHBT. Fascia lata autograft harvested from ipsilateral thigh was folded and securely sutured. The folded fascia graft was then introduced through standard anterolateral portal and fixed to superior glenoid superiorly and greater tuberosity laterally with suture anchors. Anteriorly, fascia lata graft was sutured with proximal portion of LHBT instead of subscapularis tendon. A full coverage of rotator cuff defect by facial graft was then confirmed arthroscopically to allow tension-free suturing between posterior margin of fascia graft and residual infraspinatus tendon. Postoperative abduction brace and regular follow-up was arranged.

\section{Results}

Being intact in 4 patients and either partially torn or subluxed in 14 patients, LHBT in all 18 patients were available for augmentation surgery. Preliminary functional survey revealed encouraging outcomes with a mean follow-up of 11.5 months. Nine patients with more one year follow-up regained full forward elevation; ASES improved from 24.3 to 90.2. Acromiohumeral distance improved from $3.3 \mathrm{~mm}$ to $8.6 \mathrm{~mm}$ with radiographic analysis in those 9 patients.

\section{Conclusions}

Arthroscopically LHBT augmented SCR is a novel technique and can be one of feasible opinions for irreparable rotator cuff tear.

\section{Background}

Given tremendous advances in arthroscopic techniques, treatment of massive rotator cuff tear (RCT) remains a challenge to shoulder surgeons. In case that the tear was irreparable or too extensive to have an optimal repair, the rotator cuff muscle became stiffer and atrophy with fatty infiltration. The absence 
of rotator cuff integrity and coordination leads to proximal migration of humeral head with a loss of balanced force couple [1]. As the condition is progressed, the shoulder becomes significantly painful, diminished range of motion and functional disabling [2].

Clinical presentations of irreparable RCT include chronic pain, functional limitation, weak external rotation, and even pseudoparalysis [3, 4].

Superior capsule reconstruction (SCR) is a new technique and has been currently introduced to treat irreparable RCT by using tensor fascia lata (FL) autograft [5] or human dermal (HD) allograft [6-8]. Both showed encouraging short-term outcomes. SCR was originally designed by Mihata et al. Biomechanical study showed the interposed patch graft completely restored superior stability in the cadaveric shoulder with massive RTC when the graft was securely attached to the superior glenoid tubercle and sutured to the torn tendons [9]. Despite encouraging outcome in recent reports, debates still exist regarding options and fixation of the graft [10] and the importance of marginal convergence [11]. Owing to proximal availability, long head of biceps tendon (LHBT) has been used to support RCT repair [12]. In this paper, we described an arthroscopic technique of LHBT augmentation in autologous FL SCR for irreparable RCTs and to report the preliminary outcomes.

\section{Methods}

\section{Statement of ethics approval and description of patient data}

Ethics committee approval was obtained from our Institutional Review Board for this study. We conducted a retrospective analysis in 18 patients, who underwent the index surgery between October 2017 and November 2019. There were 9 male and 9 female patients with an average of 66.1 years (range, 57 to 73). Time interval of exaggerating disability with the presentation of painful drop arm averaged 6.1 months (range, 2 to 12) according to patients' description.

\section{Preoperative Survey}

Eligible indications for performing the index surgery are irreparable rotator cuff tear with Goutallier stage 2 to 4 muscle fatty infiltration in the preoperative radiographs. The retracted supraspinatus tendon was confirmed to be irreparable during shoulder arthroscopy as the torn tendon cannot reach to the original footprint [5]. Infraspinatus tendon was completely or partially torn and subscapularis tendon was either intact or only partial articular-side tear. An existing LHBT either intact or partially torn was used and incorporated with FL graft for reconstruction of superior joint capsule. Before surgery, all patients singed informed consents for surgery and underwent complete assessment including shoulder physical examination, plain radiographs and magnetic resonance image (MRI) for confirmation of diagnosis and surgical indications.

\section{Surgical Technique}


Under general anesthesia, the patient was placed in lateral decubitus position. Longitudinal skin incision was made over lateral thigh of the same side with the lesioned shoulder. Tensor FL graft of about $4 \times$ $15 \mathrm{~cm}$ in size was harvested under meticulous dissection and hemostasis. Then the wound was approximated layer by layer with subcutical suture. After proper dressing, the patient was changed to beach-chair position. Standard arthroscopic portals was used including posterior, posterolateral, lateral, anterolateral, anterior, and Neviaser portals. A meticulous examination was undertaken to confirm the irreparable RCTs, the availability of LHBT, the condition of subscapularis tendon and articular cartilage (Figure one). For the articular-sided or upper full-thickness subscapularis tear, approximation with $2.3 \mathrm{~mm}$ Iconix all-suture anchor (Stryker Endoscopy, San Jose CA) was performed at the tuberosity footprint. Then the arthroscope was introduced into the subacromial space through posterior portal. A motorized shaver and a radiofrequency device (Super TurboVac 90; ArthroCare, Texas, USA) were used for thorough bursectomy, release of tendon adhesion, and debridement of tendon stump on the greater tuberosity through anterolateral and posterolateral working portals. Acromioplasty was not undertaken routinely unless remarkable anterolateral spur was found and removed by the motorized shaver. Further preparation of supraglenoid fossa and greater tuberosity to facilitate graft-bone healing was performed by a 4.0-mm motorized burr. In most cases, degenerative changes or partial detachment was noted over superior labrum. Motorized shavers were used for trimming and refreshing the detached edge of the superior labrum.

After measuring the optimal location and proper in-between distance, two 1.4-mm Iconix all-suture anchors (Stryker Endoscopy, San Jose CA) were inserted on the supraglenoid fossa after measuring the proper distance between two anchors (Fig. 2A and 2B).The FL graft was folded and securely sutured to become a $3 \times 4 \mathrm{~cm}^{2}$ patch with thickness about 6 to $8 \mathrm{~mm}$ by following the principles and recommendations in Mihata's mechanical study [10]. Since the FL graft patch was thicker than HD allograft, an extended skin incision of $2 \mathrm{~cm}$ was made along the lateral portal from acromion margin downward to facilitate the passage of the thicker graft into the joint. A 2-cm cannula was inserted through deltoid muscle splitting. With the shoulder in $30^{\circ}$ forward flexion and $30^{\circ}$ abduction position and supported stably in an arm holder, The FL graft patch was then introduced through the anterolateral portal. The two 1.4-mm Iconix all-suture anchors on the supraglenoid fossa were used to fix the medial 3$\mathrm{cm}$ side of the graft patch. The lateral portion of the graft patch was then secured to the greater tuberosity in compression suture-bridging technique using two 2.3-mm Iconix all-suture anchors (Stryker Endoscopy, San Jose CA) and two 4.5-mm Reelx anchors (Stryker Endoscopy, San Jose, CA) (Fig. 2C and 2D). Then proximal part of LHBT was sutured to the anterosuperior part of fascia graft (Fig. 3) while the distal part was left undetached. Before suturing the fascia graft to residual infraspinatus posteriorly, the integrity and of graft patch and full coverage of the rotator cuff defect was meticulously confirmed with arthroscopic examination (Video). Then the wounds were closed with 3-0 Nylon suture. Abduction pillow brace was applied for arm immobilization in $45^{\circ}$ abduction position.

\section{Postoperative Care}


The operation arm was immobilized in $45^{\circ}$ abduction brace for 4 weeks. Then the rehabilitation program started with Codman exercise and gentle assisted forward elevation. Active motion was prohibited until full passive elevation was reached, and began at least 4 months after SCR surgery. For patients with inadequate internal rotation, passive stretch was started at least 6 months after surgery. Resistant exercise was allowed at 9 months postoperatively.

\section{Preliminary Results}

Under arthroscopic examination, LHBT was available to augmentation surgery in all 18 patients. LHBT was intact in 4 patients and grossly intact but subluxed in 9 patients; in the remaining 5 patients, LHBT was partially torn but yet grossly sufficient to serve as an augmentation and allow suturing to FL graft. Postoperative follow-up averaged 11.5 months (range, 6 to 24); 9 patients were more than one year; 5 patients, 9 months; 4 patients, 6 months. In the 9 patients of more than one year follow, residual soreness was only noted in one patient with daily activity; ASES improved from 24.3 (range, 18 to 53) preoperatively to 90.2 (range, 82 to 96 ). All 9 patients regained full forward elevation with either no or slight limitation of internal rotation. Radiographic assessment (Fig. 4) by two of the co-authors in the those 9 patients revealed acromiohumeral distance increased from $3.3 \mathrm{~mm}$ (range, 0 to 6.3 ) to $8.6 \mathrm{~mm}$ (range, 5.1 to 9.3). Owing to limited sample size and short-term follow-up, no correlation analysis or statistical test was performed.

\section{Discussion}

Owing to disruption of shoulder force couple, absence of rotator cuff and superior capsule may change mechanics of shoulder joint with subsequent upper migration of glenohumeral articulation [13, 14]. The technique of SCR was designed to provide a biologic constrain to superior migration and thus to obtain a stable fulcrum of humeral head $[15,16]$. Autogenous $F L$ and allogenic HD patch are two common grafts used for SCR to achieve encouraging outcome. In this report, FL autograft is chosen for SCR because it is readily available and patients do not have any payment for the graft patch. Other advantages with FL autograft may include thicker spacing, less elongation $[10,17]$ and no concerns in immune responses. Potential disadvantages may include long scar and wound pain along the lateral thigh. Whether any longterm side effect would contest the clinical application may need subsequent investigation and follow-up.

In addition, we incorporate LHBT into FL graft for SCR. The rationales are threefold. Firstly, LHBT is regionally available inside the shoulder joint and has been successfully used as augmentations for supporting biomechanical forces in rotator cuff repair $[12,18]$. Incorporation of LHBT with FL patch may provide additional anterior-superior thickness and stabilizing effect against anterior translation. Secondly, FL patch could be secured antero-superiorly by suturing to LHBT instead of subscapularis tendon since anterior side-to-side suture may not be necessary or even arise concerns of shoulder stiffness owing to closure of rotator interval [17]. Thirdly, LHBT is rich in tenocytes that may imitate the physiological demands of humeral head stabilizers and is thus considered as a suitable graft for augmentation in RCT repair and reconstruction $[18,19]$. 
Currently, LHBT was considered as a suitable and locally available graft for SCR [20]. The intraarticular portion of LHBT was cut distally and fixed to greater tuberosity by either suture anchors [20-22] or transosseous suture [23]. While LHBT was used to bridge superior glenoid and greater tuberosity similar to FL in SCR, general concerns in those techniques are regarding the size and quality of tendon graft. LHBT in patients with massive RCT is commonly found to be partial torn or degenerative [24], which is not able to be accurately evaluated before arthroscopy. Instead of using LHBT alone for SCR, we incorporate LHBT to FL autograft for augmentation. By preservation of LHBT insertion and continuity, not only the incorporated LHBT could further secure the FL patch and stabilize the humeral head by creating a downward force [21] but also may provide better vascularity for graft healing [25]. In spite of being traditionally considered a pain generator, none of our patients exhibited residual anterior shoulder pain owing to biceps tenosynovitis after surgery. Further image survey was arranged at one-year follow-up.

The critical concern of this technique remains on the availability and quality of LHBT. Even though degenerative change and partial tear are commonly seen in massive RCT, our technique could be applied whenever LHBT is available. It is because LHBT is incorporated to FL patch and preservation of tendon vascularity and continuity may enhance tendon-fascia healing. Being different from other techniques using LHBT alone for SCR surgery, our method confirms availability of LHBT from preoperative MRI and thus can be readily planned before surgery. Other limitations and disadvantages include possible pain generator of LHBT, additional suture tangling for LHBT-FL suture, and technically demanding in passing FL patch to superior glenoid in the presence of LHBT. Short term follow-up in our patients as well as surgical outcomes in other reports may minimize the consideration of LHBT complications in serving as a graft for SCR. While incorporation of LHBT to FL patch may create more suture tangling, this technique does not need additional suture anchor fixation and thus skip the possible complications with suture anchor and poor bone quality. Given a steep learning curve, the index surgery demands disciplined team work similar to the original SCR technique as well as other arthroscopic reconstruction procedures in the shoulder. Surgeons would be recommended in readily shifting to open procedures on facing any unpropitious condition.

\section{Conclusions}

With the advantages in augmenting antero-superior glenohumeral stability while skipping anterior side-toside suture to avoid potential stiffness, we believe the proposed technique by incorporating LHBT with FL autograft for SCR can be one of useful options in irreparable massive RCT.

\section{Abbreviations}

RCT

massive rotator cuff tear

SCR

superior capsule reconstruction

$\mathrm{FL}$ 
fascia lata

HD

human dermal

LHBT

long head of biceps tendon

MRI

magnetic resonance image

ASES

American Shoulder and Elbow Society

\section{Declarations}

Ethics Approval and Consent to participate:Institutional review board approval (IRB no. 202000604B0) from Chang Gung Medical Foundation was obtained to perform a review of patient records and radiographs. Informed consent was obtained from all patients.

Consent for publication: Not Applicable.

Availability of supporting data:The datasets generated during the current study are available from the corresponding author on reasonable request.

Competing interests: No benefits in any form have been received or will be received from a commercial party related directly or indirectly to the subject of this article. The authors report no competing interests.

Funding:This research did not receive any specific grant from funding agencies in the public, commercial, or not-for-profit sectors.

Authors' contributions: In this study, ACYC is the single surgeon for all cases and responsible for study design. $\mathrm{CHH}$ is contributed to data collection and major writing of this article. $\mathrm{CHC}$ and $\mathrm{KYH}$ contributed to statistical analysis and manuscript review. CHC and CJW assisted in radiographic assessment. YSC together with ACYC is contributed to arthroscopic data reading. All authors read and approved the final manuscript.

Acknowledgements: Not applicable

\section{Authors' information}

Chen-Heng Hsu, MD; Chih-Hao Chiu, MD/PhD; Chun-Jui Weng, MD; Kuo-Yau Hsu, MD; Yi-Sheng Chan, MD; Alvin Chao-Yu Chen, MD

Institution: Bone and Joint Research Center, Department of Orthopaedic Surgery

Chang Gung Memorial Hospital-Linkou \& University College of Medicine; Taiwan, R.O.C 


\section{References}

1. Burkhart SS.A stepwise approach to arthroscopic rotator cuff repair based on biomechanical principles. Arthroscopy. 2000;16(1):82-90.

2. Dines DM, Moynihan DP, Dines J, McCann P. Irreparable rotator cuff tears: what to do and when to do it; the surgeon's dilemma. J Bone Joint Surg Am. 2006;88(10):2294-2302.

3. Khair MM, Gulotta LV. Treatment of irreparable rotator cuff tears. Curr Rev Musculoskelet Med. 2011;4(4):208-213.

4. Tokish JM, Alexander TC, Kissenberth MJ, Hawkins RJ. Pseudoparalysis: a systematic review of term definitions, treatment approaches, and outcomes of management techniques. J Shoulder Elbow Surg. 2017 Jun;26(6):e177-e187.

5. Mihata T, Lee TQ, Watanabe C, Fukunishi K, Ohue M, Tsujimura T, Kinoshita M. Clinical results of arthroscopic superior capsule reconstruction for irreparable rotator cuff tears. Arthroscopy. 2013 Mar;29(3):459-70.

6. Tokish JM, Beicker C. Superior capsule reconstruction technique using an acellular dermal allograft. Arthrosc Tech. 2015;23;4(6):e833-839.

7. Burkhart SS, Denard PJ, Adams CR, Brady pc, HartzlerRU. Arthroscopic Superior Capsular Reconstruction for Massive Irreparable Rotator Cuff Repair. Arthrosc Tech. 2016; 5(6): e1407-e1418.

8. Narvani AA, Consigliere P, Polyzois I, Sarkhel T, Gupta R, Levy O. The "pull-over" technique for arthroscopic superior capsular reconstruction. Arthrosc Tech. 2016;19;5(6):e1441-e1447.

9. Mihata T, McGarry MH, Pirolo JM, Kinoshita M, Lee TQ. Superior capsule reconstruction to restore superior stability in irreparable rotator cuff tears: a biomechanical cadaveric study. Am J Sports Med. 2012;40(10):2248-2255.

10. Mihata T, McGarry MH, Kahn T, Goldberg I, Neo M, Lee TQ. Biomechanical Effect of Thickness and Tension of Fascia Lata Graft on Glenohumeral Stability for Superior Capsule Reconstruction in Irreparable Supraspinatus Tears. Arthroscopy. 2016;32(3):418-26.

11. Shambaugh BC, Morales H, Ross G. Posterior interval tear after superior capsule reconstruction: a case report. J Shoulder Elbow Surg. 2018;27(7):e225-e229.

12. Neviaser JS. Ruptures of the rotator cuff of the shoulder: new concepts in the diagnosis and operative treatment of chronic ruptures. Arch Surg. 1971;102(5):483-485.

13. Nimura A, MD, Kato A, Yamaguchi K, Mochizuki T, Okawa A, Sugaya H, Akita K. The superior capsule of the shoulder joint complements the insertion of the rotator cuff. J Shoulder Elbow Surg. 2012;21(7):867-872.

14. Dyrna F, Kumar NS, Obopilwe E, Scheiderer B, Comer B, Nowak M, Romeo AA, Mazzocca AD, Beitzel Knut. Relationship between deltoid and rotator cuff muscles during dynamic shoulder abduction: a biomechanical study of rotator cuff tear progression. Am J Sports Med. 2018; 46 (8):1919-1926.

15. Adams CR, DeMartino AM, Rego G, Denard PJ, Burkart SS. The rotator cuff and the superior capsule: why we need both. Arthroscopy. 2016;32(12):2628-2637. 
16. Hartlzer RU, Burkhart SS. Superior capsular reconstruction. Orthopedics. 2017;40(5):271-280.

17. MihataT, Bui CNH, Akeda $M$, Cavagnaro MA, Kuenzler M, Peterson AB, McGarry MH, Itami $Y$, Limpisvasti O, Neo M, MD, Lee TQ. A biomechanical cadaveric study comparing superior capsule reconstruction using fascia lata allograft with human dermal allograft for irreparable rotator cuff tear. J Shoulder Elbow Surg. 2017;26(12):2158-2166.

18. Veen EJD, Stevens M, Diercks RL. Biceps Autograft Augmentation for Rotator Cuff Repair: A Systematic Review. Arthroscopy. 2018;34(4):1297-1305.

19. Pietschmann MF, Wagenhäuser MU, Gülecyüz MF, Ficklscherer A, Jansson V, MüllerPE. The long head of the biceps tendon is a suitable cell source for tendon tissue regeneration. Arch Med Sci. 2014;10(3):587-596.

20. Boutsiadis A, Chen S, Jiang C, Lenoir H, Delsol P, Barth J. Long Head of the Biceps as a Suitable Available Local Tissue Autograft for Superior Capsular Reconstruction: "The Chinese Way". Arthrosc Tech. 2017;6(5):e1559-e1566.

21. Kim YS, Lee HJ, Park I, Sung GY, Kim DJ, Kim JH. Arthroscopic In Situ Superior Capsular Reconstruction Using the Long Head of the Biceps Tendon. Arthrosc Tech. 2018;7(2):e97-e103.

22. Kim D, Jang Y, Park J, On M. Arthroscopic Superior Capsular Reconstruction With Biceps Autograft: Snake Technique. Arthrosc Tech. 2019;8(10):e1085-e1092.

23. Chillemi C, Mantovani M, Gigante A. Superior capsular reconstruction of the shoulder: the $A B C$ (Arthroscopic Biceps Chillemi) technique. Eur J Orthop Surg Traumatol. 2018;28(6):1215-1223.

24. Dimock RAC, Malik S, Consigliere P, Imam MA, Narvani AA. Superior Capsule Reconstruction: What Do We Know? Arch Bone Jt Surg. 2019;7(1):3-11.

25. Hana F, Kong CH, Hasanb MY, Ramruttunc AK, Kumar VP. Superior capsular reconstruction for irreparable supraspinatus tendon tears using the long head of biceps: A biomechanical study on cadavers. Orthop Traumatol Surg Res. 2019; 105(2):257-263.

\section{Supplemental Legends}

Video. Intraoperative video clip using 30凸Arthroscope through standard posterior portal in right shoulder of 67 year-old female patient. Firstly, the posterior margin of fascia lata graft above exhibited tension-free overlap with the residual rotator cuff tissue below. By advancing the arthroscope anteriorly and then laterally, fascia graft is found to be securely fixed on greater tuberosity with compression suture bridge technique. Thus, full coverage of rotator cuff defect by fascia graft is confirmed. The arthroscope is then moving toward anterior-superior aspect where the long head of biceps tendon (LHBT) is identified and probed above the fascial lata graft. The proximal portion of LHBT is secured to the fascial lata graft by ethibond sutures.

The <medial $>$ means glenoid side; <latera $>$, greater tuberosity side. 


\section{Figures}

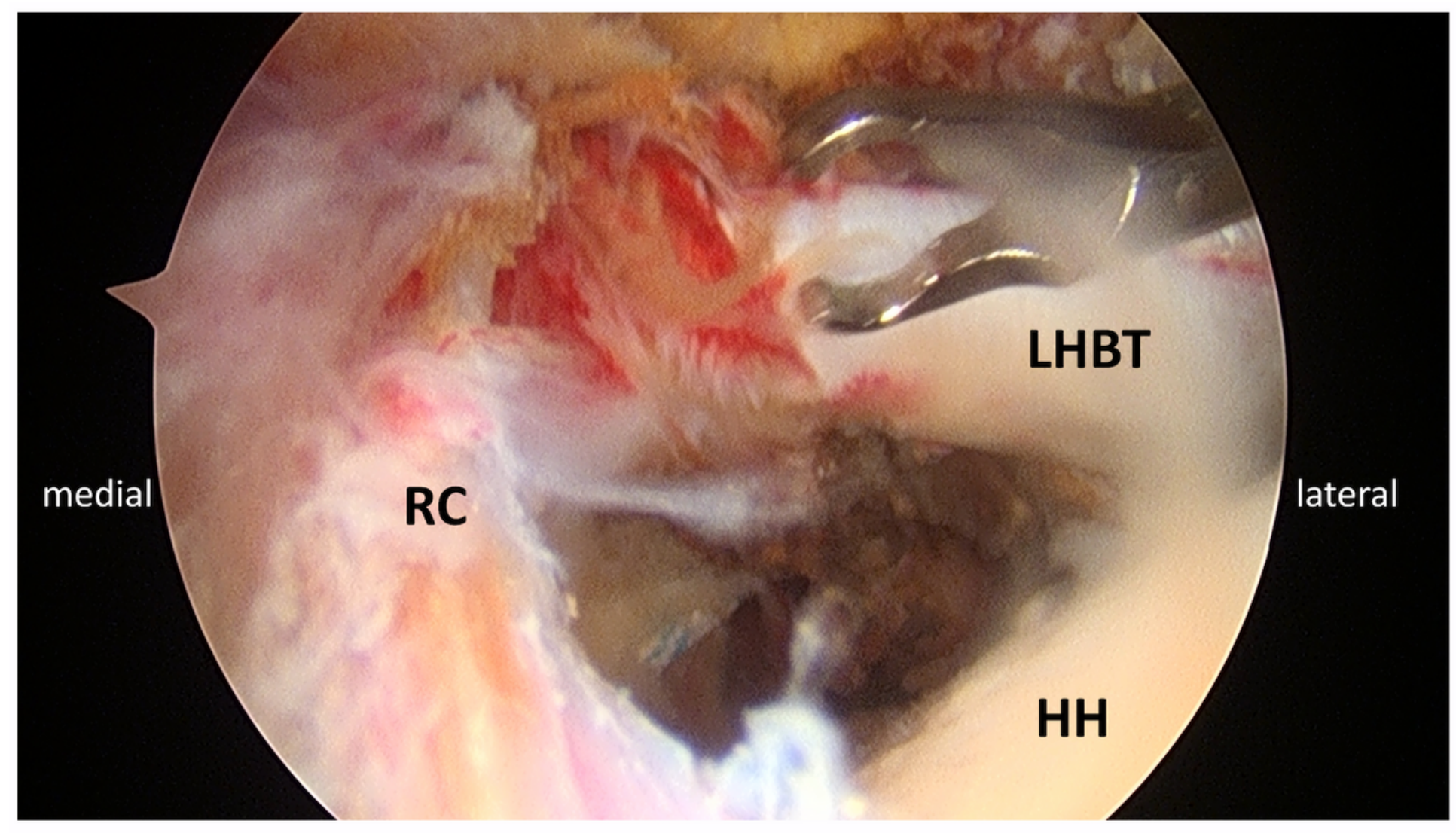

\section{Figure 1}

A $30^{\circ}$ Arthroscope is introduced via standard posterior portal in right shoulder of 67 year-old female patient with identification of residual rotator cuff tear (RC), grossly intact but medially subluxed long head of biceps tendon (LHBT) and humeral head (HH). 


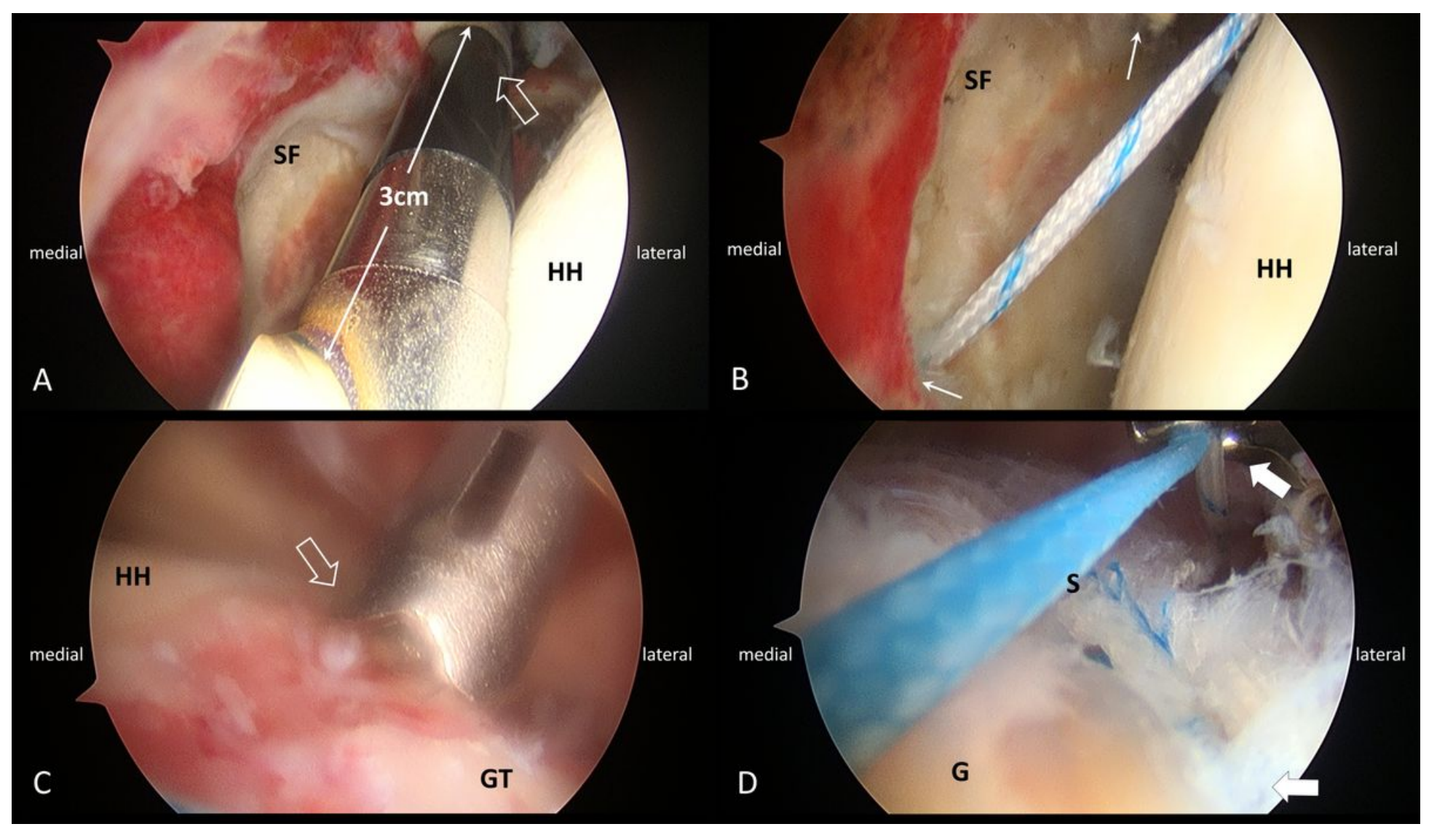

\section{Figure 2}

A $30^{\circ}$ Arthroscope is introduced via standard posterior portal in right shoulder of the same patient. [A] A radiofrequency device is inserted from anterior portal to measure the width of supraglenoid fossa (SF) for locating the insertion points for two separate Iconix suture anchors. The length from the white mark (white hollow arrow) to the device tip is $3 \mathrm{~cm}$. HH: humeral head. [B] Two $1.4 \mathrm{~mm}$ Iconix suture anchors are applied to the supraglenoid fossa (SF). [C] Predrilling (white hollow arrow) before $2.3 \mathrm{~mm}$ anchor insertion medially to greater tuberosity (GT). [D] Suture (S) shuffling for compression suture-bridging fixation of the lateral portion of the fascia lata graft $(G)$. 


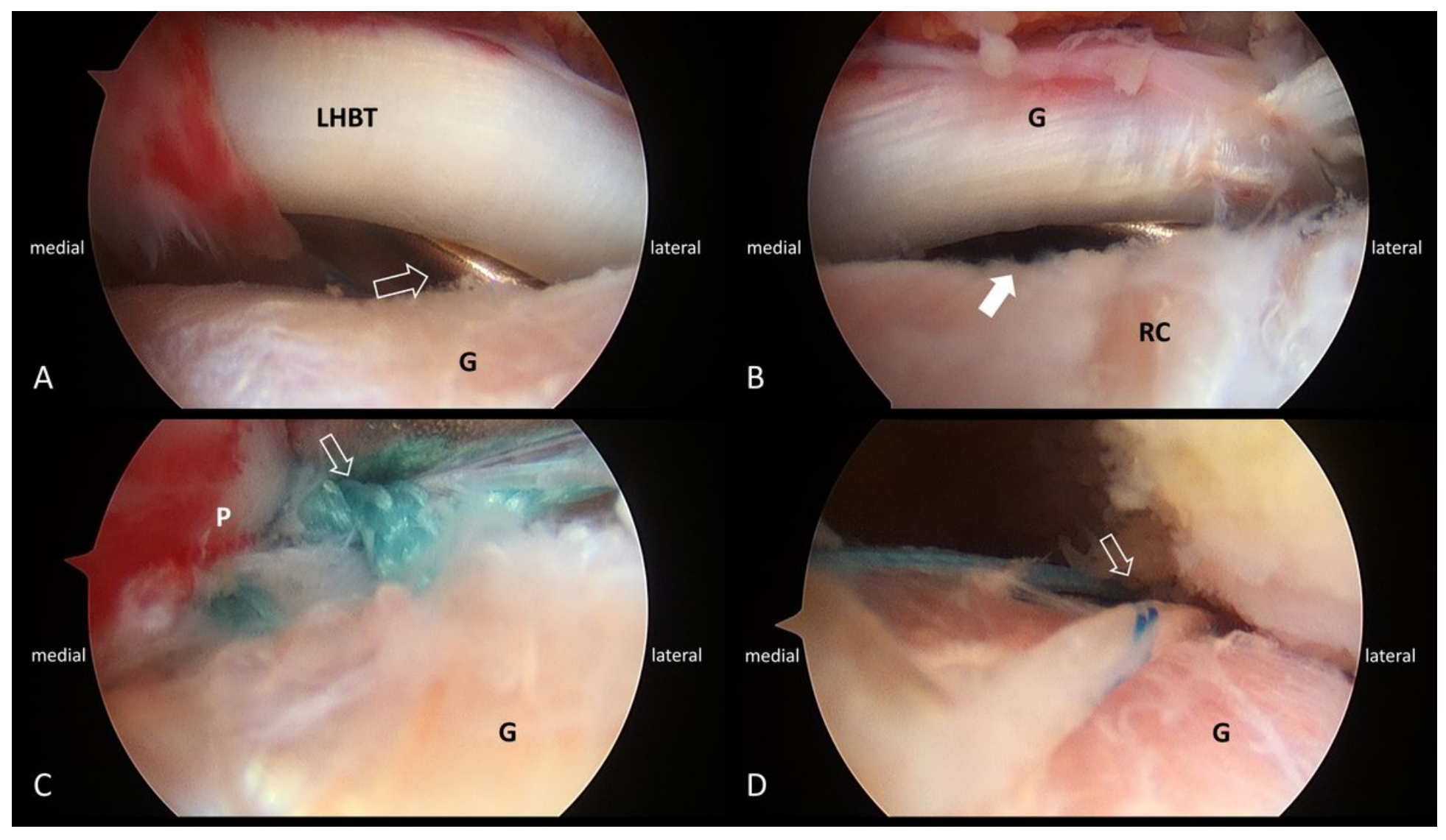

\section{Figure 3}

A $30^{\circ}$ Arthroscope is introduced via standard posterior portal in right shoulder of the same patient. [A] Anteriorly, identifying long head of biceps tendon (LHBT) above the fascia graft (G) by probing (hollow arrow). [B] Posteriorly, confirming optimal size and location of fascia graft $(\mathrm{G})$ to fully cover the rotator cuff defect and reach the margin (white arrow) of residual rotator cuff tissue (RC). [C] Proximal part of the LHBT $(P)$ is then sutured (hollow arrow) the fascia graft (G). [D] Laterally, fascial graft (G) is fixed to graft tuberosity using compression suture bridging technique (hollow arrow). 


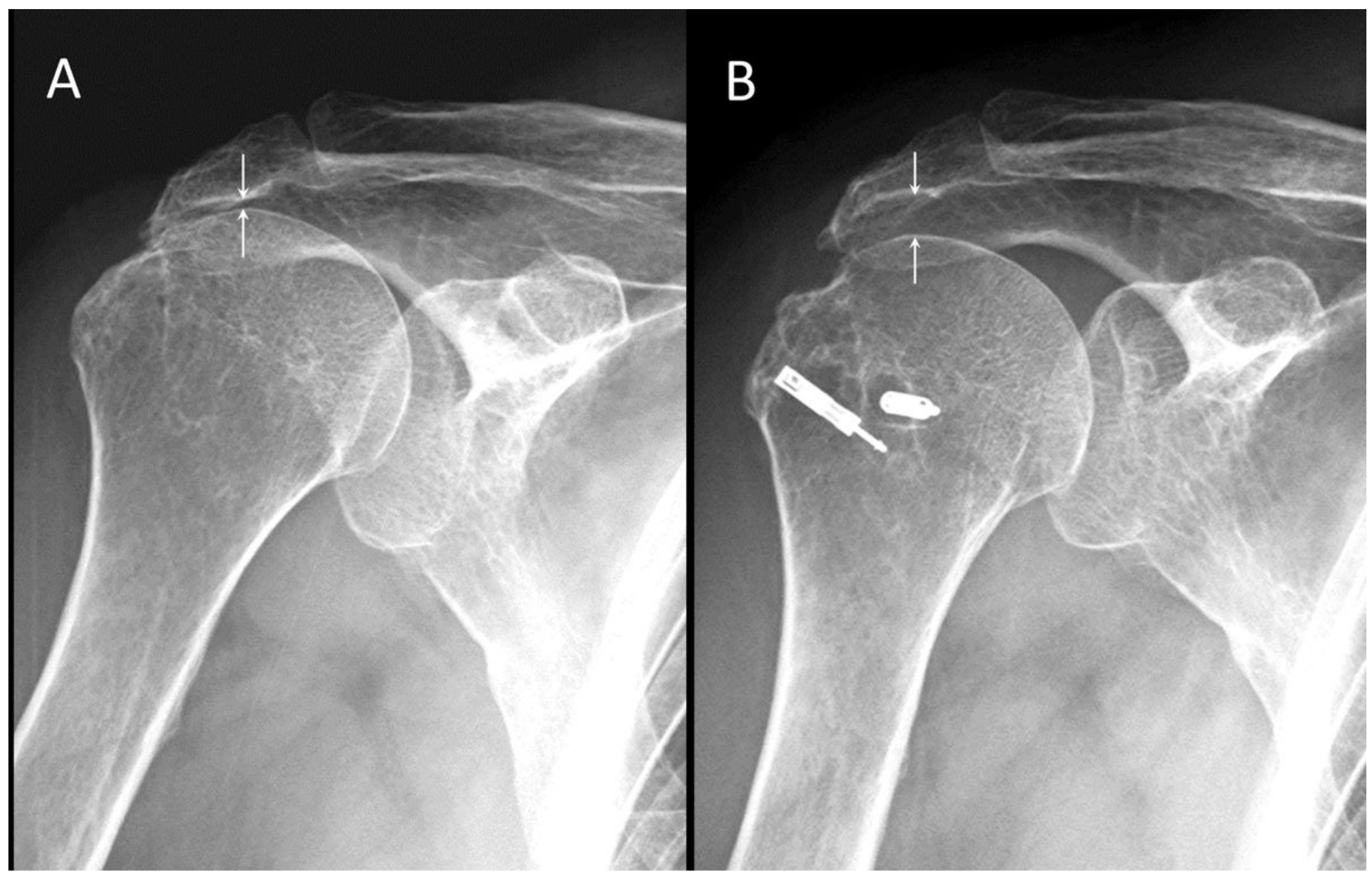

\section{Figure 4}

Radiographic assessment for acromiohumeral distance (white arrows). [A] Before surgery. [B] One year after surgery.

\section{Supplementary Files}

This is a list of supplementary files associated with this preprint. Click to download.

- SCRvideooriginal.mp4 